\title{
Effect of cholesterol on structural and mechanical properties of membranes depends on lipid chain saturation
}

\author{
Jianjun Pan, ${ }^{1}$ Stephanie Tristram-Nagle, ${ }^{1}$ and John F. Nagle ${ }^{1,2, *}$ \\ ${ }^{1}$ Department of Physics, Carnegie Mellon University, Pittsburgh, Pennsylvania 15213, USA \\ ${ }^{2}$ Department of Biological Sciences, Carnegie Mellon University, Pittsburgh, Pennsylvania 15213, USA
}

(Received 11 May 2009; published 25 August 2009)

\begin{abstract}
The effects of cholesterol on membrane bending modulus $K_{\mathrm{C}}$, membrane thickness $D_{\mathrm{HH}}$, the partial and apparent areas of cholesterol and lipid, and the order parameter $S_{\text {xray }}$ are shown to depend upon the number of saturated hydrocarbon chains in the lipid molecules. Particularly striking is the result that up to $40 \%$ cholesterol does not increase the bending modulus $K_{\mathrm{C}}$ of membranes composed of phosphatidylcholine lipids with two cis monounsaturated chains, although it does have the expected stiffening effect on membranes composed of lipids with two saturated chains. The $B$ fluctuational modulus in the smectic liquid crystal theory is obtained and used to discuss the interactions between bilayers. Our $K_{\mathrm{C}}$ results motivate a theory of elastic moduli in the high cholesterol limit and they challenge the relevance of universality concepts. Although most of our results were obtained at $30{ }^{\circ} \mathrm{C}$, additional data at other temperatures to allow consideration of a reduced temperature variable do not support universality for the effect of cholesterol on all lipid bilayers. If the concept of universality is to be valid, different numbers of saturated chains must be considered to create different universality classes. The above experimental results were obtained from analysis of x-ray scattering in the low angle and wide angle regions.
\end{abstract}

DOI: 10.1103/PhysRevE.80.021931

PACS number(s): 87.16.dm, 87.14.Cc, 87.64.Bx

\section{INTRODUCTION}

Cholesterol is an important component of mammalian cell membranes [1]. It is considered to be a key molecule involved in the lateral in-plane heterogeneity in lipid bilayers [2]. It plays a central role in the sometimes contentious [3] raft concept that is a major emphasis in the study of biomembranes [4]. A recent review, with a focus on simulations, emphasizes that determining and understanding the interactions of cholesterol with different lipids is challenging and unresolved [5].

Our recent letter [6] reported that the effect of cholesterol on lipid bilayer properties depends on the number of saturated chains in the lipids. Our most striking result was the comparison of how cholesterol affects the bending modulus of lipids with different numbers of saturated hydrocarbon chains. When both chains are saturated, the bending modulus $K_{\mathrm{C}}$ increases dramatically with added cholesterol. On the other hand, when both chains are monounsaturated (one cis $\mathrm{C}=\mathrm{C}$ double bond per chain), $K_{\mathrm{C}}$ remains essentially the same up to cholesterol mole fraction $c=0.4$. There have been other observations that are consistent with the effect of cholesterol being dependent upon chain saturation. Micropipette aspiration measurements reported that with the addition of $50 \%$ cholesterol the elastic area stretch modulus $\left(K_{\mathrm{A}}\right)$ increased by sixfold for SOPC, a lipid with one saturated (the " $\mathrm{S}$ " for stearoyl) chain and one monounsaturated (the "O" for oleoyl) chain, while there was only a threefold increase for DOPC, a lipid that has two unsaturated $\mathrm{O}$ chains, at $15{ }^{\circ} \mathrm{C}[7]$. Wide angle $\mathrm{x}$-ray scattering (WAXS) results have suggested that the thermal behavior of DOPC is different from that of DMPC, with two (D) saturated myristoyl (M)

\footnotetext{
*Corresponding author; nagle@cmu.edu
}

chains, and SOPC as cholesterol is added. As the temperature is increased in bilayers of pure lipids, there is an abrupt increase in the width of the WAXS peak at the chain melting main transition temperature $T_{\mathrm{M}}[8]$. As the cholesterol concentration $c$ is increased, the change in peak width for both DMPC and SOPC becomes spread over a wider temperature range. In contrast, for DOPC a sharp transition has been reported to persist even at $c=0.52$ [9]. In a similar manner, the peak shape of the differential scanning calorimetry endotherm also broadens and finally disappears for both DMPC and SOPC when the cholesterol concentration is increased $[10,11]$, but the sharp endotherm has been reported to persist for DOPC even for cholesterol mole fraction $c=0.5$ [11]. However, it may be noted that the transition temperature of DOPC at $-17{ }^{\circ} \mathrm{C}$ is considerably below the freezing temperature of ice which could obfuscate the interpretation. The rotational diffusion of androstane spin-label was reported to decrease much more when $30 \%$ cholesterol was added to DMPC than to DOPC [12]. The chemical potential of cholesterol is larger in saturated lipid bilayers [13] and it partitions more favorably into vesicles of saturated lipid than vesicles of unsaturated lipid [4]. This latter review emphasizes that cholesterol interacts differently with lipids with different headgroups.

While there have been many indications that the effect of cholesterol depends upon hydrocarbon chain saturation, nevertheless, it has been emphasized [14] that most studies on the effect of cholesterol on lipid bilayers used saturated lipids and these results have formed the conventional wisdom, outside of a cadre of cholesterol cognoscenti, regarding the effect of cholesterol on lipid bilayers (in contrast to the well recognized partitioning of cholesterol in lipid mixtures or rafts). A recent exception which did not employ saturated lipids compared the effect of different sterols, but used only the POPC lipid, and that led to the proposal that the effect of 
sterols on lipid bilayers is universal [15]. In contrast to both the conventional wisdom and to the universality concept, our results emphasize that the effect of cholesterol is very different for different lipid bilayers having different numbers of saturated hydrocarbon chains.

Section II in this paper describes our experiments and how we analyze our data. Section III reports results from low angle x-ray scattering (LAXS) for bilayer thickness $D_{\mathrm{HH}}$ and for two different definitions of the area of lipid and cholesterol molecules. Section IV reports in-plane spacing $d$ and order parameter $S_{\text {xray }}$ from WAXS. Section V presents results for the bending modulus $K_{\mathrm{C}}$ and Sec. VI reports the bulk modulus $B$ and its use with $K_{\mathrm{C}}$ in discussing interactions between bilayers. Section VII presents data taken at different temperatures to address whether universality can be recovered by considering temperature relative to the main chain melting transition temperature $T_{\mathrm{M}}$. A general discussion is given in Sec. VIII regarding why cholesterol affects the physical properties of lipid bilayers differently when the lipids have different numbers of saturated chains.

\section{SAMPLES AND X-RAY SCATTERING}

\section{A. Lipids}

Synthetic DOPC (1,2-dioleoyl-sn-glycero-3phosphocholine), SOPC (1-stearoyl-2-oleoyl-sn-glycero-3phosphocholine), DMPC (1,2-dimyristoyl-sn-glycero-3phosphocholine), DPPC (1,2-dipalmitoyl-sn-glycero-3phosphocholine), and diC22:1PC (1,2-dierucoyl-snglycerol-3-phosphocholine) were purchased in lyophilized form from Avanti Polar Lipids (Alabaster, AL) and used as received. Intimately mixed samples of cholesterol and lipid were made by dissolving the individual components in chloroform, mixing in the appropriate mole fractions $c$ $=N_{\text {Chol }} /\left(N_{\text {lipid }}+N_{\text {Chol }}\right)$, and then evaporating the organic solvent using a KNF filtration pump (Trenton, NJ).

\section{B. Unilamellar vesicles}

Following our usual procedure [16], about $10 \mathrm{mg}$ of pure lipid or lipid/cholesterol mixtures was added to $500 \mu \mathrm{l}$ milli-Q water in a nalgene plastic vial and then hydrated by repetitive cycles of heating and cooling between $50{ }^{\circ} \mathrm{C}$ and $-20{ }^{\circ} \mathrm{C}$ with vortexing until the multilamellar vesicles (MLV) were uniformly dispersed. The unilamellar vesicles (ULV) sample was obtained by passing the MLV through an Avanti miniextruder 25 times, using polycarbonate filters with $500 \AA$ diameter pores. The average size of the ULV sample was about $600 \AA$ in diameter, as obtained by light scattering and neutron scattering of similarly prepared vesicles [17]. ULV samples were $\mathrm{x}$ rayed using a Rigaku RUH3R microfocus rotating anode (Woodlands, TX) equipped with a Xenocs FOX2D focusing collimation optic at the power of $40 \mathrm{kV} \times 100 \mathrm{~mA}$. The $\mathrm{x}$-ray wavelength was $1.5418 \AA\left(\mathrm{Cu} \mathrm{K}_{\alpha}\right)$, the sample to detector (Rigaku Mercury CCD) distance was $660 \mathrm{~mm}$, and a flight path filled with helium was used between the sample and the detector. Pure water scattering was subtracted from the ULV sample scattering.

\section{Oriented multilamellar samples}

About $200 \mu$ l chloroform/methanol (2:1, v/v) was added to $\sim 4 \mathrm{mg}$ samples which were then plated onto $30 \times 15$ $\times 1 \mathrm{~mm}$ silicon wafers within a glove box and a stack of $\sim 2000$ oriented lipid bilayers was formed for each sample using the rock and roll procedure [18]. The sample on the wafer was then trimmed to a $30 \times 5 \mathrm{~mm}$ strip after drying at least for one day in the glove box and another day in a fume hood. Samples were $x$ rayed on two runs at the CHESS G1 station, where the wavelengths $(\lambda=1.2742 \AA$ or $1.1803 \AA)$ were obtained using double bounce multilayer monochromators. The data were collected with a Flicam charge coupled device (CCD) (pixel size $0.0698 \mathrm{~mm}$ ) with sample to CCD distance 399.7 or $370.9 \mathrm{~mm}$ for LAXS and 154.7 or 149.1 $\mathrm{mm}$ for WAXS. Each X-ray exposure ranged from 20 to $60 \mathrm{~s}$ and the sample was moved laterally after a few minutes of exposure to keep radiation damage negligible. Lipid breakdown, examined by thin layer chromatography after x-ray exposure, was $<0.1 \%$. The flat sample was rotated from $-3^{\circ}$ to $7^{\circ}$ continuously in $\theta$ relative to the beam in the LAXS experiment. In the WAXS experiment, it was fixed at $\theta$ $=0.2^{\circ}$ for sample collection and $\theta=-0.2^{\circ}$ for background collection. Some of the WAXS data were also taken by using the in-house rotating anode. Hydration through the vapor was facilitated with the help of a Peltier cooler under the sample to lower the temperature of the sample relative to the water vapor $\left(<0.1^{\circ} \mathrm{C}\right)$, thereby condensing water onto the sample. The final lamellar $D$ spacing was within a few angstroms of that obtained from unoriented MLV in excess water.

\section{Analysis of LAXS}

To obtain electron density profiles (EDP) of lipid bilayers, we first obtain the Fourier transform $\left|F\left(q_{z}\right)\right|$ from our scattering data. For oriented multilamellar samples (ORI) samples, the form factor $F\left(q_{z}\right)$ is related to the scattering intensity by $I(\mathbf{q})=S(\mathbf{q})\left|F\left(q_{z}\right)\right|^{2} / q_{z}$, where $S(\mathbf{q})$ is the structure factor and $q_{z}$ is the Lorentz correction for oriented samples. In order to obtain $S(\mathbf{q})$, smectic liquid crystalline theory is applied to fit the $q_{r}$ dependence of the background subtracted diffuse scattering intensity $[19,20]$. The fitting obtains the two material parameters, the bending modulus $K_{\mathrm{C}}$ and the compression modulus $B$. Using these two moduli, the theoretical structure factor $S(\mathbf{q})$ was constructed and divided into $I(q)$ to obtain a preliminary $\left|F\left(q_{z}\right)\right|^{2} / q_{z}$ which was then corrected for absorption and to account for the fact that the average thickness projected onto $z$ of an undulating bilayer is larger than if the bilayer is perfectly flat [21]. The $q$ range of the form factor obtained from ORI data is from $\sim 0.2 \AA^{-1}$ to $\sim 0.85 \AA^{-1}$. ULV samples were also used to obtain data at smaller $q_{z}$. The form factor for ULV is related to the scattering intensity by $I(\mathbf{q})=\left|F\left(q_{z}\right)\right|^{2} / q_{z}^{2}$, where $q_{z}^{2}$ is essentially the Lorentz correction for isotropic powder samples and the structure factor $S(\mathbf{q})$ is assumed to be one for dilute samples and for $q_{z}$ values larger than $0.01 \AA^{-1}$ [22]. Assuming that the small size of ULV suppresses undulations, no geometric undulation correction was applied. For most of the ULV data, because we only used a rotating anode $\mathrm{x}$-ray source, the large back- 


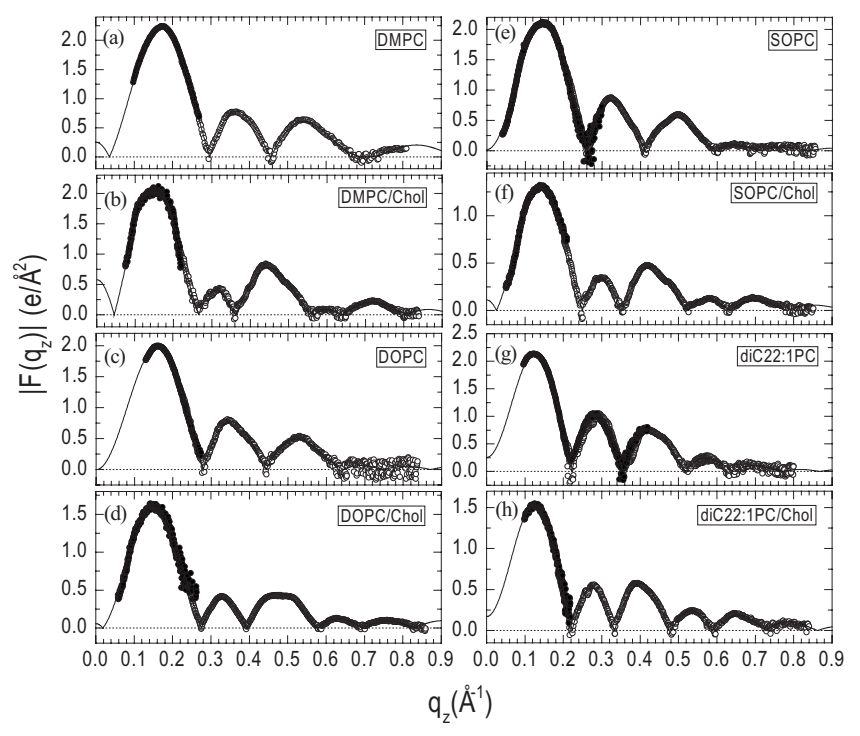

FIG. 1. H2 model fits (solid lines) to experimental form factors measured at $30{ }^{\circ} \mathrm{C}$ (open symbols from oriented ORI samples and solid symbols from ULV samples). The lipid/cholesterol mixtures shown below the pure lipids had cholesterol mole fractions $c=0.3$. The data for pure lipids were from previous papers: DMPC [75], DOPC [61], diC22:1PC [16], and SOPC [76]. When the intensity is near zero, the raw data produce Gaussian distributions of apparent amplitudes that include some negative values of $\left|F\left(q_{z}\right)\right|$ that are shown in the figure; these are included in the $\mathrm{H} 2$ fitting to avoid biasing the model to overly large values of $\left|F\left(q_{z}\right)\right|$.

ground intensity made it difficult to obtain good signal-tonoise ratio at higher $q$ values. This explains why the highest $q$ value from ULV sample is smaller than the $\sim 0.4 \AA^{-1}$ that has previously been reported [16].

\section{E. Analysis of WAXS}

Wide angle $\mathrm{x}$-ray scattering data were obtained for oriented cholesterol/lipid mixtures. The wide angle $d$ spacing was determined as $2 \pi / q_{\max }$ where the intensity in the $q_{r}$ direction was largest at $q_{\max }$. The order parameter $S_{\text {xray }}$ $=\left(3\left\langle\cos ^{2} \beta-1\right\rangle\right) / 2$ was calculated [23] from the angular distribution of the WAXS scattering intensity $I(\varphi)$, where $\beta$ is the angle between the bilayer normal and the scattering entities, which are often supposed to be chains, although there is also scattering involving cholesterol. A chain order parameter $S_{\text {mol }}$ is usually measured by deuterium NMR. Because $S_{\text {mol }}$ for unsaturated lipids is difficult to obtain using NMR, and because $S_{\text {xray }}$ has been shown to track the NMR order parameter quite well as a function of cholesterol concentration for the saturated lipid DPPC [23], $S_{\text {xray }}$ appears to be an appropriate substitute for NMR order parameters.

\section{STRUCTURE FROM LAXS}

Figure 1 shows some obvious differences between the form factors for lipids with and without cholesterol. The first noticeable difference is that cholesterol makes the magnitude and the width of the second lobe smaller and the lobes at higher $q$ more prominent. The second difference is that cho-

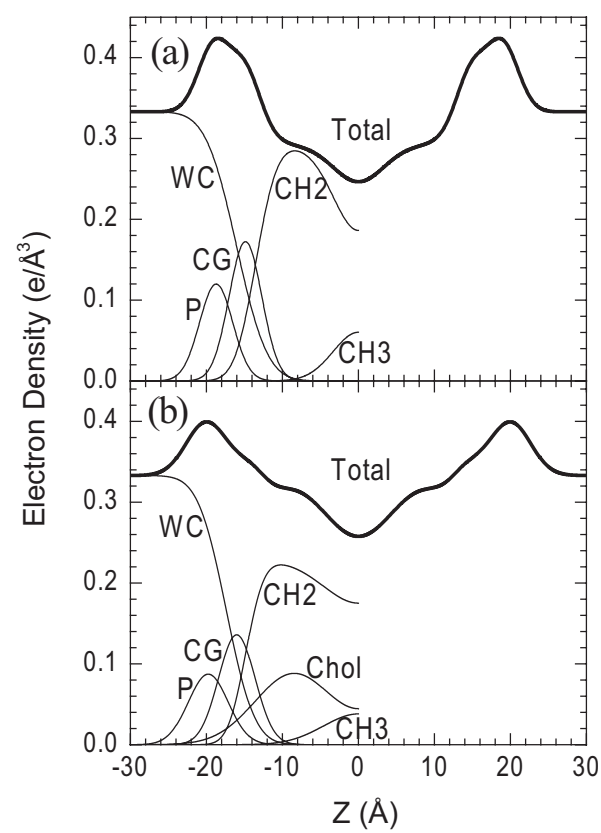

FIG. 2. Total electron density profiles of DOPC with cholesterol mole fractions (a) $c=0$ and (b) $c=0.3$ versus distance $z$ from the center of the bilayer are shown as the thick curves labeled total. The contributions from the individual lipid components and cholesterol in the $\mathrm{H} 2$ model are shown only for negative values of $z$ with thinner lines labeled WC (water plus choline), (p) phosphate, (CG) carbonyl plus glycerol, $\left(\mathrm{CH}_{2}\right)$ methylene plus methine groups on the hydrocarbon chains, $\left(\mathrm{CH}_{3}\right)$ terminal methyl groups, and (Chol) cholesterol.

lesterol moves the positions of the local minima to smaller $q$ values, suggesting thicker bilayers, as is confirmed by the EDP that we subsequently obtain.

The EDP and various structural parameters are obtained by fitting the $\mathrm{H} 2$ model [24] to the experimental form factor. In the model, a lipid bilayer is divided into several components, including (i) water plus choline, (ii) phosphate, (iii) glycerol plus carbonyls, (iv) methylenes and methines on the chains, and (v) terminal methyl groups on the chains. Volume data [25] and two assumptions, the volume ratio $r=2$ of terminal methyl to average methylene group [26] and the distance $D_{\mathrm{H} 1}=4.95 \AA$ between the phosphate and the Gibbs dividing surface of the hydrocarbon region [27], were also applied. With added cholesterol, the EDP of the cholesterol component was modeled as a Gaussian distribution function. The fits of this model to some of the experimental $\left|F\left(q_{z}\right)\right|$ are shown in Fig. 1.

The total EDP and the electron density of the component groups inferred from the modeling are shown in Fig. 2 for DOPC with and without $30 \%$ cholesterol. The magnitude of the largest total electron density, which occurs at a $z$ value near the average position of the electron dense phosphate group, decreases because cholesterol "dilutes" the lateral density of phosphate groups. From the EDP, we extracted the lipid bilayer thickness $D_{\mathrm{HH}}$, which is defined to be the distance between the two maxima in the EDP. The fit has about $90 \%$ of the electron density of cholesterol located within the hydrocarbon region, but, unlike the robust result for $D_{\mathrm{HH}}$, the precise location of cholesterol should not be considered to be 


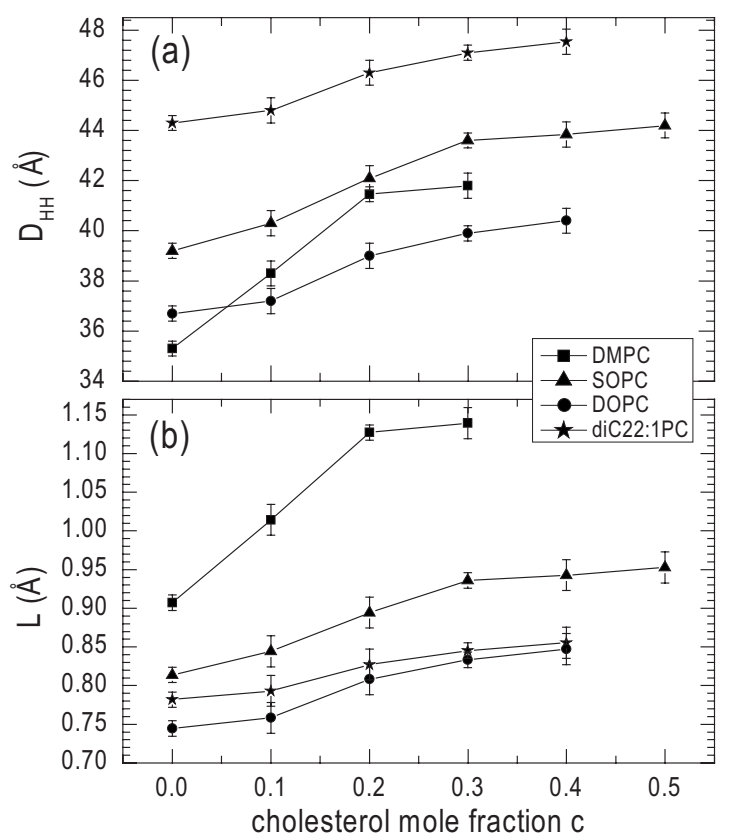

FIG. 3. (a) Bilayer thickness $D_{\mathrm{HH}}$ versus cholesterol mole fraction $c=N_{\text {Chol }} /\left(N_{\text {lipid }}+N_{\text {Chol }}\right)$. The $D_{\text {HH }}$ values for DOPC at $c=0.1$, 0.2 , and 0.4 are from [77]. (b) Average travel of the chain methylenes along the bilayer normal $L=D_{\mathrm{C}} /\left(n_{\mathrm{CH}_{2}}+r\right)$.

a definitive result in view of the overparametrization of the model compared to the available experimental data. Different molecular-dynamics simulations suggest different hydrogen bonded associations with the hydroxyl headgroup of cholesterol [5], but our results are not accurate enough to discriminate between them. In the future, cholesterol simulations should be compared directly to our experimental form factors $\left|F\left(q_{z}\right)\right|$, as is being done for single component lipid bilayers [24,28-31].

Our results for $D_{\mathrm{HH}}$ for all of the lipids investigated are shown in Fig. 3(a). The $D_{\mathrm{HH}}$ for DMPC increases rapidly as $c$ increases to 0.2 and it levels off at higher $c$. The first $10 \%$ cholesterol has a much smaller effect on $D_{\mathrm{HH}}$ for DOPC and diC22:1PC. The behavior of $D_{\mathrm{HH}}$ is very similar for DOPC and $\mathrm{diC} 22: 1 \mathrm{PC}$ even though the latter has four more $\mathrm{CH}_{2}$ groups on each chain and the cis double bond position is located at C13-C14 instead of C9-C10 in DOPC. This is very similar to the result in [32] that the bilayer thickness of diC14:1PC and diC22:1PC increases by almost the same amount with the addition of $40 \%$ cholesterol. Hung et al. [33] reported an increase of $6.7 \AA$ for DMPC at $c=0.3$, which agrees very well with our $6.5 \AA$ increase. Similar increases in the Luzzati thickness $D_{\mathrm{B}}$ with cholesterol concentration have been reported in neutron-scattering studies on unilamellar vesicles of DMPC at temperatures above the main transition temperature of DMPC [34,35]. Our values of $D_{\mathrm{HH}}$ for DOPC and diC22:1PC at different $c$ also agree with other reported values $[33,36,37]$.

The comparison of $D_{\mathrm{HH}}$ for the different lipids in Fig. 3(a) is suboptimal because some lipids, especially diC22:1PC, have more carbons/chain and that difference dominates the comparison of the overall $D_{\mathrm{HH}}$. More revealing is Fig. 3(b) which plots the average travel $L$ of each carbon along the $z$

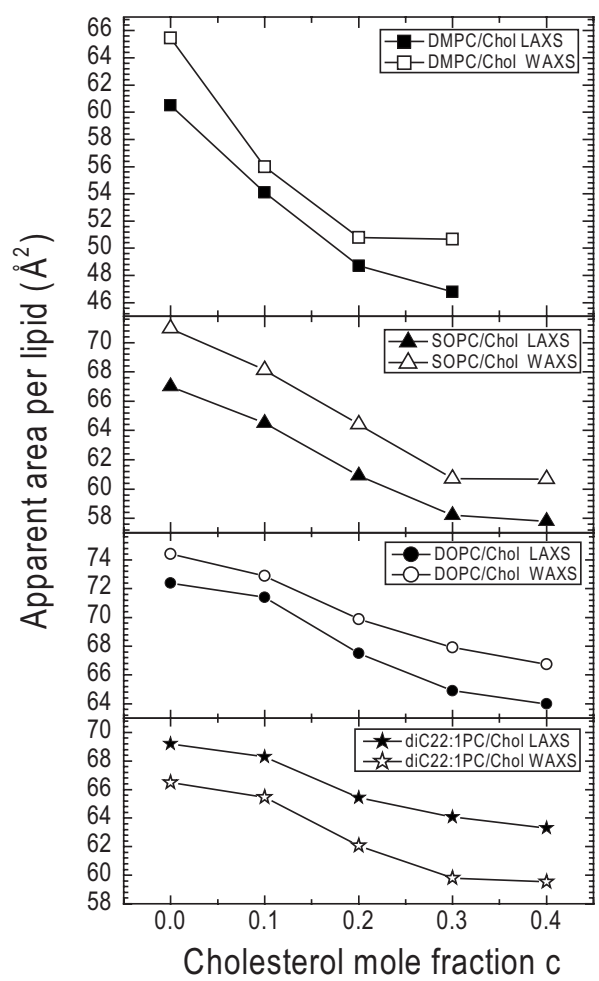

FIG. 4. Apparent area per lipid molecule $A_{\text {Lapp }}$ calculated from LAXS (solid symbols) and WAXS (open symbols) versus cholesterol mole fraction $c$.

axis. $L$ is calculated as $D_{\mathrm{C}} /\left(n_{\mathrm{CH}_{2}}+r\right)$, where $D_{\mathrm{C}}$ is half the hydrocarbon thickness (vide infra) and where $n_{\mathrm{CH}_{2}}$ is the number of $\mathrm{CH}_{2}$ groups plus the number of double bonded $\mathrm{CH}$ groups on each hydrocarbon chain and the $r$ term with $r=2$ accounts for the greater effective length of the terminal $\mathrm{CH}_{3}$ groups. Figure 3(b) shows more clearly than Fig. 3(a) that the behavior of SOPC with one saturated and one unsaturated chain is intermediate between DMPC with both chains saturated and DOPC and diC22:1PC with both chains unsaturated.

We calculated the hydrocarbon thickness $D_{\mathrm{C}}$ from $D_{\mathrm{HH}}$ $=2\left(D_{\mathrm{C}}+D_{\mathrm{H} 1}\right)$ using our values of $D_{\mathrm{HH}}$ and the value $D_{\mathrm{H} 1}$ $=4.95 \AA$ from previous studies [27]. (However, note that a recent study suggests a smaller $D_{\mathrm{H} 1} \sim 3.9 \AA$ for DOPC [38].) From $D_{\mathrm{C}}$, an apparent area per lipid $A_{\text {Lapp }}$ and an apparent area per cholesterol $A_{\text {Cholapp }}$ were calculated using

$$
A_{\text {Lapp }}=\left(V_{\mathrm{L}}-V_{\mathrm{H}}\right) / D_{\mathrm{C}} \text { and } A_{\text {Cholapp }}=f V_{\text {Chol }} / D_{\mathrm{C}},
$$

where the values of $V_{\text {Chol }}$ were taken from the measured partial molecular volume of cholesterol [25], $f$ is the fraction of the cholesterol in the region 0 to $D_{\mathrm{C}}$ obtained from the $\mathrm{H} 2$ model fitting, $V_{\mathrm{L}}$ is the partial molecular volume per lipid [25], and $V_{\mathrm{H}}=331 \AA^{3}$ is the headgroup volume (consisting of the phosphate, choline, glycerol, and carbonyl groups) which was obtained from gel phase DMPC [27]. The results for $A_{\text {Lapp }}$ are plotted in Fig. 4. The calculation of the apparent areas in Eq. (1) assumes that both cholesterol and lipid molecules have average cylindrical shapes with the same cross-sectional area in the entire hydrocarbon region 
from $0<z<D_{\mathrm{C}}$. This is clearly not the case for cholesterol which has a larger area in its ring region and negligible area near the center of thick bilayers, so the apparent areas are, at best, average areas that do not take into account the detailed packing of the two types of molecules in a bilayer. This explains that our results for $A_{\text {Cholapp }}$ are larger for thinner bilayers (averaging $36 \AA^{2}$ for DMPC and $38 \AA^{2}$ for DOPC) and smaller for thicker bilayers $\left(31 \AA^{2}\right.$ for $\operatorname{diC} 22: 1 \mathrm{PC}$ and $34 \AA^{2}$ for SOPC).

It has been previously emphasized that the definition of molecular areas in the simulation literature has been subject to arbitrary definitions [39], but that there is a unique thermodynamic definition of area based on the concept of partial specific volumes in physical chemistry [39] that we here apply to experimental data. In this paper we call the latter quantities the partial molecular volumes and the corresponding quantities for area by the name partial molecular area, $A_{\text {Lpar }}$ for lipid and $A_{\text {Cholpar }}$ for cholesterol. Generally, partial molecular areas $A_{i, \mathrm{par}}(c)$ are defined as the derivative of the total area $A_{\text {Tot }}$ with respect to the number, $A_{i, \text { par }}(c)$ $=\left(\partial A_{\text {Tot }} / \partial N_{i}\right)_{N j, j \neq i}$ and then homogeneous function theory proves that $A_{\mathrm{Tot}}=\Sigma_{i} N_{i} A_{i \text {, par }}(c)$, where only one variable $c$ suffices for the fully hydrated binary mixtures in this study.

Obtaining partial molecular areas from experimental data first uses the $\mathrm{H} 2$ fitting program to obtain the area per average molecule $A_{\mathrm{N}}=A_{\mathrm{Tot}} /\left(N_{\mathrm{L}}+N_{\mathrm{Chol}}\right)$ as

$$
A_{\mathrm{N}}=\left[(1-c)\left(V_{\mathrm{L}}-V_{\mathrm{H}}\right)+c f V_{\mathrm{Chol}}\right] / D_{\mathrm{C}} .
$$

$A_{\mathrm{N}}$ is just the mole weighted sum of the apparent areas in Eq. (1), but it contains no assumption regarding the lateral spatial distribution between cholesterol and lipid. Following the procedure described in [39], the results for $A_{\mathrm{N}}$ were fitted to an analytical function and the partial molecular areas were obtained by extrapolating the slope of the curve at each concentration $c$ to $c=0$ to obtain $A_{\mathrm{Lpar}}(c)$ and by extrapolating to $c=1$ for $A_{\text {Cholpar }}(c)$. Results for partial molecular area are shown in Fig. 5 for DMPC and for DOPC. As was the case for simulations of DPPC/cholesterol mixtures [39], $A_{\text {Cholpar }}$ for DMPC has a strongly negative value for small $c$ due to the condensing effect of cholesterol on neighboring saturated hydrocarbon chains. We emphasize that this result, which may be surprising to readers not familiar with negative values of experimental partial molecular volume, is not only correct, but also understandable because a single cholesterol induces a straightening of many lipid hydrocarbon chains so that the total area can be decreased rather than increased [39]. The partial molecular area of DMPC remains high at small $c$ because an additional lipid does not change the number of strongly affected near-neighbor lipids and therefore it can be thought of as being added to a region of the bilayer that is relatively unperturbed by cholesterol. As $c$ increases, $A_{\text {Lpar }}$ approaches its more perturbed value and $A_{\text {Cholpar }}$ approaches a value closer to the values that are obtained from geometric ways of estimating cholesterol area in simulations.

Figure 5 shows that $A_{\text {Cholpar }}$ also displays the condensing effect for DOPC, but less so than for DMPC as its value at $c=0$ is positive, though small. This is consistent with cholesterol affecting fewer lipid chains in DOPC compared to DMPC as reported in [6]. Also, $A_{\text {Lpar }}$ decreases less than for

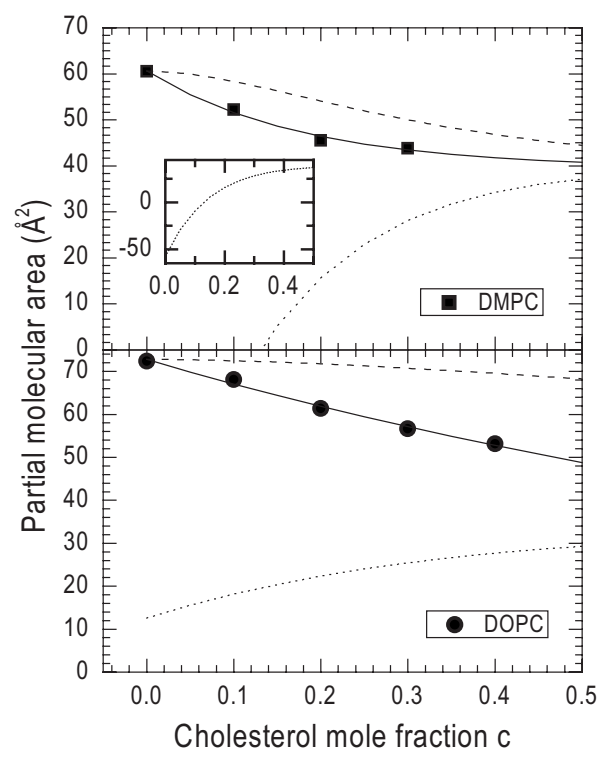

FIG. 5. Partial molecular area of lipid $A_{\text {Lpar }}$ and cholesterol $A_{\text {Cholapp }}$ versus cholesterol mole fraction $c$ for DMPC and DOPC. The data points $A_{\mathrm{N}}$ obtained from Eq. (2) and the $\mathrm{H} 2$ model fit to the x-ray form factors. The solid lines are fits based on Eq. (12) in [39] to area per average molecule in Eq. (2). The dashed lines are the partial molecular areas of lipid obtained from the intercept at $c=0$ of the tangent to the black curve and the dotted lines (also in the inset for DMPC) are the partial molecular areas of cholesterol obtained from the intercept at $c=1$.

DMPC as $c$ increases. The $c$ dependences of the partial molecular areas for diC22:1PC (not shown) are very similar to those of DOPC in Fig. 5. The $c$ dependences of the partial molecular areas for SOPC behave in an intermediate fashion between disaturated DMPC and the dimonounsaturated lipids, with a slightly negative value of $A_{\text {Cholpar }}$ at $c=0$ and a value at $c=0.5$ between that of DMPC and that of the dimonounsaturated lipids. Also, the decrease in $A_{\text {Lpar }}$ for SOPC as $c$ increases is intermediate between the more rapid decrease for DMPC and the more gradual decrease for the lipids with two monounsaturated chains.

It may also be mentioned that the extrapolation of the solid curves in Fig. 5 to $c=1$ provides estimates for the bare area of cholesterol $A_{\text {Cholbare }}$ as shown in the Appendix of [39]. The results are $39.3 \AA^{2}$ for $A_{\text {Cholbare }}$ in DMPC, $35.3 \AA^{2}$ in SOPC, $31.7 \AA^{2}$ in DOPC, and $28.9 \AA^{2}$ in diC22:1PC, which follows the trend with degree of saturation rather than the trend with thickness that is followed by $A_{\text {Cholapp. }}$ It is sometimes desired to estimate a single value for $A_{\text {Cholbare }}$ in all bilayers at all concentrations. We obtain such an estimate, $A_{\text {Cholbare }}=32.6 \AA^{2}$, by fitting all the $A_{\mathrm{N}}$ data points while requiring the extrapolated value at $c=1$ (namely, $A_{\text {Cholbare }}$ ) to be the same for all four lipids. However, we note that this estimate would be different if we had studied a different set of lipids.

One conclusion from this section is that there is no single cholesterol area in lipid bilayers. The best defined partial molecular $A_{\text {Cholpar }}$ depends strongly on concentration, and our apparent $A_{\text {Cholapp }}$ and our $A_{\text {Cholbare }}$ values depend upon the lipid. This conclusion is consistent with the lateral variations that must occur when the different flexible chains from 


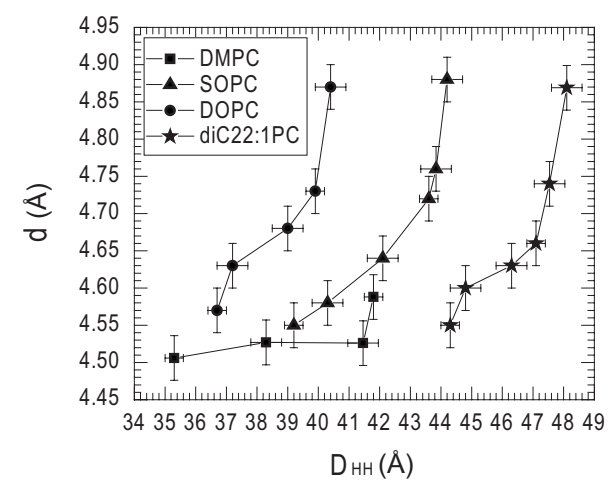

FIG. 6. Wide angle $d$ spacing versus bilayer thickness $D_{\mathrm{HH}}$.

different lipids interact with cholesterol that also has a shorter flexible chain and whose rigid ring structure also has a tilting degree of freedom. Nevertheless, for those who wish a single number, our choice would be $33 \AA^{2}$ obtained in the preceding paragraph by requiring $A_{\text {Cholbare }}$ to fit all the lipids we studied. This value may be compared to the area of $36.2 \AA^{2}$ obtained for cholesterol monohydrate crystals [40].

\section{RESULTS FROM WAXS}

The wide angle $d$ spacing as a function of $c$ has been reported many times $[8,9,23,41]$. Here we plot it against the bilayer thickness $D_{\mathrm{HH}}$ in Fig. 6, where the smaller values of $D_{\mathrm{HH}}$ correspond to the lower concentrations $c$ of cholesterol as shown in Fig. 3(a). At low $c, d$ increases as the bilayer becomes thicker, but the increase is smaller for DMPC than for the other three lipids. The slopes in Fig. 6 increase dramatically at high $c$ because $D_{\mathrm{HH}}$ levels off at high concentrations, as expected, because most chains have already been affected by becoming neighbors of cholesterol, while the average $d$ continues to increase. We attribute this continued increase in $d$ to spatial correlations involving cholesterol (both with lipids and with other cholesterols) and not just to spatial correlations between pairs of hydrocarbon chains. These correlations involving cholesterol also scatter $\mathrm{x}$ rays in addition to the scattering from lipid-lipid chain correlations, so it is not warranted to conclude that the chain-chain $d$ spacing increases at high $c$.

Both experiment and simulation have shown that incorporation of cholesterol increases lipid chain orientational order in the fluid phase by straightening the chains and ordering them along the bilayer normal $[15,23,42-45]$. Our chain order parameter $S_{\text {xray }}$ as a function of cholesterol concentration from WAXS analysis also shows this trend. Figure 7 shows that $S_{\text {xray }}$ increases for all lipids as $c$ increases from 0 and $S_{\text {xray }}$ levels off at higher $c$. (Note that at even higher concentrations that we chose not to study, cholesterol reaches a solubility limit of $c_{\max }=0.66$ in phosphatidylcholine lipids and then crystallizes into a separate phase [46].) While this general picture applies to all the lipids in Fig. 7, there are differences between lipids with different numbers of saturated chains. The initial slope $\left(d S_{\text {xray }} / d c\right)_{c=0}$ is the largest for DMPC and DPPC with two saturated chains. It is the smallest for DOPC and diC22:1PC with two monounsaturated

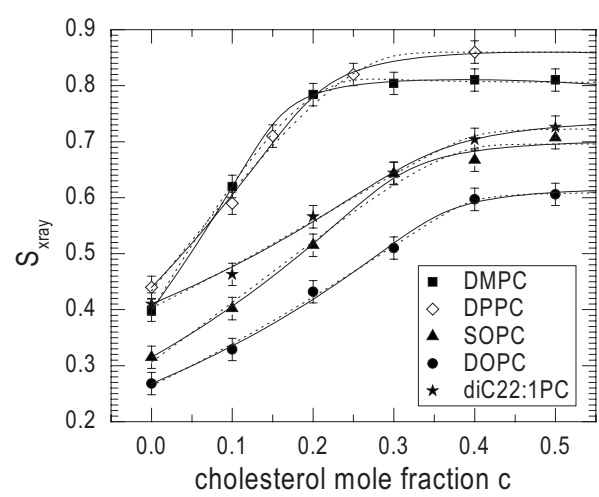

FIG. 7. Symbols show order parameter $S_{\text {xray }}$ from WAXS versus cholesterol mole fraction. The solid curves show the fits to the model: free cholesterol $+p$ free lipids $\Leftrightarrow$ complex [50]. The dotted lines show the fits to a refined model that also includes perturbation caused by the complex to the free lipid $[6,33]$.

chains and intermediate for SOPC. Furthermore, at the high cholesterol end, $S_{\text {xray }}$ for DMPC and DPPC levels off near $c \sim 0.2$ while it still increases for the other lipids, only leveling off for SOPC, DOPC, and diC22:1PC near $c \sim 0.4$. A similar progression of the NMR chain order parameter as a function of sterol concentration has also been detected in DPPC/cholesterol [42], DPPC/ergosterol [47], and POPC/ ergosterol $[15,48,49]$ systems. The leveling off of the NMR order parameter has been interpreted either as representing the high cholesterol end of the liquid ordered and liquid disordered phase coexistence region [47] or as representing the completion of lipid/cholesterol complex formation [50].

It is also of interest to compare the magnitude of $S_{\text {xray }}$ for the different lipids in Fig. 7. There are two general hypotheses that help to explain the trends. (1) Saturated chains have greater chain order than unsaturated chains when the chain lengths are the same; this hypothesis is consistent with cis unsaturated chains being constrained to have a disordering bend at the double bond that prevents their achieving $S_{\text {xray }}=1$. (2) Longer chains have greater order than shorter chains when the amount of unsaturation is the same; this hypothesis is motivated by longer chains having larger van der Waals cohesive interactions and less relative disruption (entropy) due to end effects. With no cholesterol, the trend in $S_{\text {xray }}$ from Fig. 7 is $\operatorname{DPPC}(16,2)>\operatorname{DMPC}(14,2)$ $\approx \operatorname{diC} 22: 1 \mathrm{PC}(22,0)>\operatorname{SOPC}(18,1)>\operatorname{DOPC}(18,0)$, where the numbers in parentheses are $(N=$ length of chain in carbons, $M=$ number of saturated chains). This trend is consistent with hypothesis (1) when only $M$ is different (SOPC vs DOPC) and consistent with hypothesis (2) when $N$ is different (DPPC vs DMPC) and (diC22:1PC vs DOPC). The first interesting comparison is DMPC with diC22:1PC which suggests that $\Delta N=8$ has an equivalent effect on $S_{\text {xray }}$ as $\Delta M=-2$. This would suggest that pairs of lipids with $(\Delta N$ $=4, \Delta M=-1$ ) would also have the same $S_{\text {xray }}$, but comparison of SOPC with DMPC shows that replacing just one saturated chain in the $s n-2$ position of DMPC while increasing the chain lengths by four has more disordering effect than a subsequent replacement of the remaining saturated chain in the $s n-1$ position of SOPC while again increasing the lengths of the chains by four to form diC22:1PC. This is consistent 
TABLE I. Elastic properties. $K_{\mathrm{C}}$ and $D_{\mathrm{C}}$ at $T=30{ }^{\circ} \mathrm{C}$ are from this paper and $K_{\mathrm{A}}$ at $T=33{ }^{\circ} \mathrm{C}$ are from [7] except for the results with superscripts.

\begin{tabular}{lccccccc}
\hline \hline Lipid & Chol $(c)$ & $\begin{array}{c}T \\
\left({ }^{\circ} \mathrm{C}\right)\end{array}$ & $K_{\mathrm{C}}\left(10^{-20} \mathrm{~J}\right)$ & $\begin{array}{c}D_{\mathrm{C}} \\
(\AA)\end{array}$ & $\begin{array}{c}K_{\mathrm{A}} \\
(\mathrm{mN} / \mathrm{m})\end{array}$ & $R$ & $\begin{array}{c}\delta \\
(\AA)\end{array}$ \\
\hline SOPC & 0.0 & $30-33$ & 8.8 & 14.6 & 290 & 1.2 & 19.1 \\
& 0.3 & $30-33$ & 13.6 & 16.8 & $430^{\mathrm{a}}$ & 1.5 & 19.5 \\
& 0.5 & $30-33$ & 13.9 & 17.1 & 1130 & 4.0 & 12.2 \\
& 0.5 & 15 & $24.6^{\mathrm{b}}$ & $17.7^{\mathrm{c}}$ & 1990 & 4.2 & 12.2 \\
DOPC & 0.0 & $30-33$ & 7.6 & 13.4 & 290 & 1.1 & 17.8 \\
& 0.3 & $30-33$ & 7.4 & 15.0 & $420^{\mathrm{d}}$ & 2.1 & 14.5 \\
& 0.5 & $30-33$ & $7.4^{\mathrm{e}}$ & 15.3 & 870 & 4.6 & 10.1 \\
& 0.5 & $30-33$ & $9.6^{\mathrm{f}}$ & 15.3 & 870 & 3.5 & 11.5 \\
\hline \hline
\end{tabular}

${ }^{\mathrm{a}}$ Calculated from $K_{\mathrm{A}}$ for $c=0.3$ at $T=15^{\circ} \mathrm{C}$ [73] using temperature dependence interpolated from $c=0$ and $c=0.5$ [7].

${ }^{\mathrm{b}}$ Obtained from [62].

${ }^{\mathrm{c}}$ Calculated from $T=30{ }^{\circ} \mathrm{C}$ using hydrocarbon thickness contractivity $\alpha_{D_{\mathrm{C}}}=\left(1 / D_{\mathrm{C}}\right)\left(\partial D_{\mathrm{C}} / \partial T\right)=0.0019 / \mathrm{deg}$ of DOPC [61].

${ }^{\mathrm{d}}$ Same as at $15{ }^{\circ} \mathrm{C}$ based on measured temperature independence for DOPC/cholesterol [7].

e Obtained from $c=0.4$.

${ }^{\mathrm{f}}$ Assuming a $30 \%$ increase for $c=0.5[74]$ compared with $c=0.4$.

with a previous report that the area per lipid $A$ is increased more by replacing one saturated chain than by the subsequent replacement of the second saturated chain [16]. At the highest cholesterol concentrations in Fig. 7, $S_{\text {xray }}$ levels off at higher values; these values follow a modified trend with different lipids $\operatorname{DPPC}(16,2)>\operatorname{DMPC}(14,2)$ $>$ diC22: 1 PC $(22,0) \sim \operatorname{SOPC}(18,1)>\operatorname{DOPC}(18,0) \quad$ which suggests that cholesterol modifies the competition between increasing $N$ and decreasing $M$. Now the comparison of $S_{\text {xray }}$ for DMPC and diC22:1PC (and SOPC) indicates that high cholesterol makes changes in $M$ relatively more important than changes in $N$. Another way to see this directly from Fig. 7 is that the increase $\Delta S_{\text {xray }}$ from $c=0$ to $c_{\text {max }}$ averages 0.42 for DMPC and DPPC, is 0.39 for SOPC, and $\Delta S_{\text {xray }}$ averages 0.33 for DOPC and diC22:1PC. This is consistent with the hypothesis that saturated chains are more susceptible to ordering by the rigid ring structure of cholesterol $[6,13,33,51,52]$.

We previously described [6] the fit of a chemical reaction model [50] to our $S_{\text {xray }}$ data in Fig. 7. The model considers an equilibrium system composed of free cholesterol, free lipid, and lipid/cholesterol complexes. The overall chain order parameter was given by

$$
S_{\text {xray }}=S_{\mathrm{F}} f_{\mathrm{F}}+S_{\mathrm{C}} f_{\mathrm{C}},
$$

where $S_{\mathrm{F}}$ and $S_{\mathrm{C}}$ are the order parameters for free lipid and lipid in complex, and $f_{\mathrm{F}}$ and $f_{\mathrm{C}}$ are the corresponding mole fractions. A further refinement included a perturbation of the free lipid by the complex using

$$
S_{\text {xray }}=\left(S_{\mathrm{F}}+\gamma f_{\mathrm{C}} / p\right) f_{\mathrm{F}}+S_{\mathrm{C}} f_{\mathrm{C}},
$$

where $\gamma$ represents the size of the perturbation [33]. Both fits are shown in Fig. 7. The results given in Table $\mathrm{I}$ in [6] indicated that cholesterol has a larger effect on lipids that have more saturated chains in two ways: (1) the larger value of $p$ obtained for saturated lipids suggests that more of them bind to one cholesterol and (2) the larger value of $\gamma$ for saturated lipids suggests that the distal chains in saturated lipids are more susceptible to ordering by cholesterol complexes [6].

Figure 4 also shows results for the area/lipid $A_{\mathrm{L}}$ obtained from WAXS using a new method of analysis [23]. The overall trend of $A_{\mathrm{L}}$ as a function of cholesterol concentration agrees between LAXS and WAXS, although the absolute values differ by a few $\AA^{2}$. $A_{\mathrm{L}}$ for DMPC starts to level off near $c \approx 0.2$ and the leveling off concentration moves to $c$ $\approx 0.3$ for SOPC and to $c \geq 0.4$ for DOPC and diC22:1. These leveling off concentrations are related to the leveling off of $S_{\text {xray }}$ in Fig. 7.

It has been traditional in the literature to calculate the hydrophobic thickness $2 D_{\mathrm{C}}$ based on a linear relationship between the thickness and the NMR chain order parameter $S_{\text {mol }}[15,43,53-55]$. We also plot our bilayer thickness $D_{\mathrm{HH}}$ as a function of the chain order parameter $S_{\text {xray }}$ in Fig. 8. A linear relationship for each lipid with different amounts of cholesterol works quite well. This is consistent with the tra-

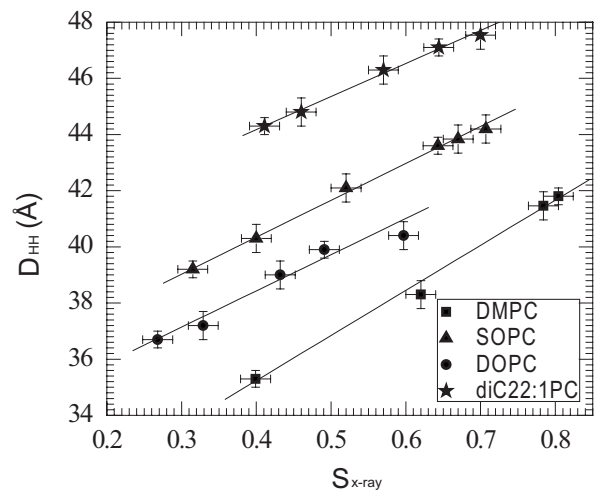

FIG. 8. Bilayer thickness $D_{\mathrm{HH}}$ versus chain order parameter $S_{\text {xray. }}$ The solid lines are linear fits for each lipid. 


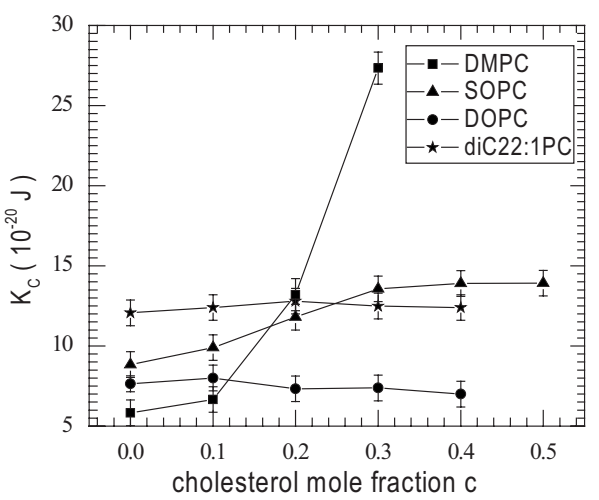

FIG. 9. Bending modulus $K_{\mathrm{C}}$ for bilayers of four lipids with cholesterol mole fraction $c$ at $30{ }^{\circ} \mathrm{C}$.

ditional NMR result because (1) Mills et al. [23] have shown that $S_{\text {xray }}$ tracks $S_{\text {mol }}$ for saturated DPPC and (2) the headhead thickness $D_{\mathrm{HH}}=2 D_{\mathrm{C}}+2 D_{\mathrm{H} 1}$ is simply related to $2 D_{\mathrm{C}}$ if one assumes that the distance $D_{\mathrm{H} 1}$ between the phosphate group and the hydrocarbon region does not change with added cholesterol. It is also interesting to extrapolate $S_{\text {xray }}$ to 1 in Fig. 8, which would correspond to chains perfectly ordered in the all-trans conformation and also aligned along the bilayer normal. This extrapolation gives $D_{\mathrm{HH}}=44.9 \AA$ for DMPC, from which $D_{\mathrm{C}}=17.5 \AA$ is obtained using $D_{\mathrm{H} 1}$ $=4.95 \AA$. This $D_{\mathrm{C}}$ is considerably larger than the measured $D_{\mathrm{C}}=15.1 \AA$ in gel phase DMPC [27] because the gel phase chains are tilted. Taking into account the chain tilt of $32.3^{\circ}$ [27], the fully extended $D_{\mathrm{C}}$ would be $15.1 \AA / \cos \left(32.3^{\circ}\right)$ $=17.9 \AA$, which is close to the extrapolated $D_{\mathrm{C}}=17.5 \AA$ above.

\section{BENDING MODULUS $\boldsymbol{K}_{\mathrm{C}}$}

An important mechanical parameter that we obtain from fitting the liquid crystalline theory to our LAXS diffuse scattering data is the bending modulus $K_{\mathrm{C}}$. Our results are shown in Fig. 9. As cholesterol concentration $c$ increases, $K_{\mathrm{C}}$ for DMPC increases rapidly and at $c=0.3$ it is already more than fourfold larger than at $c=0$. Increasing $K_{\mathrm{C}}$ decreases the intensity of the diffuse scattering required for our method, which is why we can only obtain $K_{\mathrm{C}}$ up to $c=0.3$ for DMPC. For SOPC $K_{\mathrm{C}}$ also increases, but not as dramatically as for DMPC, and above $c=0.3$ it levels off. Our results for SOPC are similar to results for POPC which has two fewer carbons in its saturated $s n-1$ chain [15]. The leveling off of $K_{\mathrm{C}}$ has also been observed for SOPC/cholesterol [56] and POPC/ ergosterol [15]. Our more surprising result is that both DOPC and diC22:1PC have essentially constant $K_{\mathrm{C}}$ for $c$ up to 0.4 . As we pointed out previously, there have been similar results scattered in the literature [6], which support the validity of our measurements. However, it had not been emphasized that the overall phenomenological description is that the effect of cholesterol on $K_{\mathrm{C}}$ increases dramatically with the number of saturated chains. Since our initial publication [6], our result that cholesterol does not increase $K_{\mathrm{C}}$ in DOPC has been thrice confirmed using entirely different techniques on giant unilamellar vesicles. Pulling tethers shows no increase for $c$ up to 0.3 [57] and up to 0.5 [58], fluctuation and electrodeformation analysis shows no increase up to $c=0.5$ [59].

At $c=0$, the sequence of $K_{\mathrm{C}}$ is $\operatorname{diC} 22: 1>$ SOPC $>$ DOPC $>$ DMPC, which is the same sequence as for the bilayer thickness $D_{\mathrm{HH}}$. Quantitatively, the polymer brush theory [60] predicts that the area modulus $K_{\mathrm{A}}$ is the same for all lipid bilayers and

$$
K_{\mathrm{C}}=K_{\mathrm{A}}\left(2 D_{\mathrm{C}}\right)^{2} / 24 \text {. }
$$

Equation (5) works well for most lipid bilayers composed of a single type of lipid $[60,61]$. This theory also accurately predicted the experimental temperature dependence of DOPC [61]. However, in its simplest form the theory does not predict the cholesterol dependence of $K_{\mathrm{A}}$, as was already indicated by the result that $K_{\mathrm{A}}$ increases upon addition of cholesterol $[7,62]$. Our result that $K_{\mathrm{C}}$ for DOPC does not increase with cholesterol directly challenges Eq. (5) because $K_{\mathrm{A}}$ and $2 D_{\mathrm{C}}$ increase for this lipid [7]. Table I shows values for $D_{\mathrm{C}}, K_{\mathrm{C}}$, and $K_{\mathrm{A}}$. The table also shows that the quantity

$$
R=K_{\mathrm{A}}\left(2 D_{\mathrm{C}}\right)^{2} / 24 K_{\mathrm{C}}
$$

deviates, as cholesterol is added, from its value close to one that it has for single component lipids.

Breakdown of the polymer brush model and Eq. (5) is not surprising as the rigid ring structure of cholesterol is hardly a polymer brush. Evans has suggested that a different model be considered for the high cholesterol concentration limit as follows. The rigid ring structure of cholesterol has a length $\delta$ of approximately $9 \AA$, which is shorter than the monolayer thicknesses $D_{\mathrm{C}}$ given in Table I. This suggests that each monolayer in the bilayer has a relatively stiff region of thickness $\delta$ and a relatively flexible region of thickness $D_{\mathrm{C}^{-}} \delta$. The general equations for the moduli are

$$
K_{\mathrm{A}}=\int \sigma(z) d \mathrm{z} \text { and } K_{\mathrm{C}}=\int \sigma(z) z^{2} d z,
$$

where $\sigma(z)=-\partial P(z) / \partial \ln [\alpha(z)], P(z)$ is the lateral pressure profile, and $\alpha(z)=\left[A(z)-A_{0}\right] / A_{0}$ is the lateral strain profile. Let us assume that $\sigma(z)$ is a constant $\sigma_{r}$ in the ring region of cholesterol in each monolayer and negligible in the nonring flexible region, which gives $K_{\mathrm{A}}=2 \delta \sigma_{r} . K_{\mathrm{C}}$ for the model is obtained by assuming that the two monolayers are uncoupled; it has been emphasized that this is a better approximation for most bilayers (those with chains of comparable length) than the traditional "unit bilayer" which assumes strong coupling between the monolayers [63]. When bending occurs, the model has a neutral plane where $\alpha\left(z_{c}\right)=0$ in the center $z_{c}$ of the ring region in each monolayer. Then, the $K_{\mathrm{C}}$ integral in Eq. (7) is performed separately for each monolayer with $z$ replaced by $z-z_{c}$. This gives

$$
K_{\mathrm{C}}=2 \int \sigma_{r}\left(z-z_{c}\right)^{2} d z=K_{\mathrm{A}} \delta^{2} / 12,
$$

which is considerably different from Eq. (6) regarding the definition of $\delta$ versus $h$, as well as the numerical coefficient. To obtain a feeling for whether this model is plausible, we use experimental values of $K_{\mathrm{C}}$ and $K_{\mathrm{A}}$ to calculate the values of $\delta$ shown in Table I. For the largest $c$, the values of $\delta$ are 
TABLE II. Quantities related to interactions between bilayers.

\begin{tabular}{|c|c|c|c|c|c|}
\hline & $\begin{array}{c}\lambda_{\mathrm{fl}} \\
(\AA)\end{array}$ & & & $H\left(10^{-21}\right.$ & J) \\
\hline \multicolumn{2}{|c|}{ Cholesterol $(c)$} & 0.0 & 0.3 & 0.0 & 0.3 \\
\hline DMPC & 3.7 & 19.8 & 14.5 & 10 & 5 \\
\hline $\mathrm{DMPC}^{\mathrm{a}}$ & 5.1 & 19.8 & 14.5 & 8 & 4 \\
\hline SOPC & 3.9 & 18.3 & 16.5 & 8 & 7 \\
\hline DOPC & 5.8 & 18.4 & 19.7 & 5 & 5 \\
\hline diC22:1PC & 4.3 & 16.8 & 18.8 & 6 & 6 \\
\hline
\end{tabular}

${ }^{\mathrm{a}}$ From [64].

reasonably close to the length $9 \AA$ of the ring structure of cholesterol and it is encouraging that $\delta$ has the same value for SOPC at two temperatures. The values of $\delta$ being larger than $9 \AA$ is consistent with the neglect of the nonring region and the larger value of $\delta$ for SOPC than for DOPC is consistent with DOPC chains being more flexible than SOPC chains as well as with the larger nonring region (larger $D_{\mathrm{C}}$ ) for SOPC. As $c$ decreases, $\delta$ becomes larger than $D_{\mathrm{C}}$, which is clearly unphysical. A more comprehensive theory of mechanical properties would require a crossover from this theory for the high cholesterol limit to the polymer brush model that applies at $c=0$.

\section{INTERACTIONS BETWEEN BILAYERS}

Table II reports our values for the steric water spacing $D_{W}^{\prime}=D-2 D_{\mathrm{C}}-18 \AA$ that are calculated from our values of $D_{\mathrm{HH}}$ and from the lamellar repeat $D$ spacing obtained from MLV samples that are immersed in water and are therefore fully hydrated by definition. The usual experimental result that there are finite nonzero values for $D_{W}^{\prime}$ even when there is excess water and no osmotic pressure pushing the bilayers together is due to competing interactions. There is an attractive van der Waals interaction that has a pressure of the form

$$
P_{\mathrm{vdW}}=\frac{H}{6 \pi}\left(\frac{1}{D_{W}^{\prime 3}}-\frac{2}{D^{3}}+\frac{1}{\left(2 D-D_{W}^{\prime}\right)^{3}}\right) .
$$

There are generally supposed to be two repulsive interactions, the hydration interaction, which becomes small at full hydration and that will be ignored, and the entropic fluctuation interaction with excess free energy compared to single bilayers,

$$
F_{\mathrm{fl}}=\left(\frac{k_{\mathrm{B}} T}{2 \pi}\right) \sqrt{\frac{B}{K_{\mathrm{C}}}},
$$

where $B$ is the modulus for the fluctuational free energy of compression in the smectic liquid crystal theory $[19,64]$. Data for $B$ for SOPC are shown in Fig. 10 as a function of the steric water spacing $D_{W}^{\prime}$. Figure 10 supports the general result $[61,65,66]$ that $B$ depends exponentially on $D_{W}^{\prime}$, which means that the repulsive fluctuation pressure $P_{\mathrm{fl}}=$ $-\left(\partial F_{\mathrm{fl}} / \partial D_{W}^{\prime}\right)_{\mathrm{T}}$ can be written as

$$
P_{\mathrm{fl}}=P_{0} \exp \left(-D_{W}^{\prime} / \lambda_{\mathrm{fl}}\right),
$$

with decay lengths $\lambda_{\mathrm{fl}}$ which are reported in Table II. The value of $\lambda_{\mathrm{fl}}$ for DOPC agrees well with an earlier result but $\lambda_{\mathrm{fl}}$ is now considerably smaller than a previous value for DMPC [64] which is also shown in Table II. Within error, the slopes of the $B$ vs $D_{W}^{\prime}$ data with cholesterol are the same in a log plot as for the pure lipid bilayer suggesting that cholesterol has little effect on $\lambda_{\mathrm{fl}}$. For SOPC and diC22:1PC the best fit straight line with $c=0.3$ cholesterol is slightly lower by a factor of 1.1 compared to no cholesterol. For DMPC the $c=0.3$ cholesterol line is lower by a factor of 1.9 whereas for DOPC, the cholesterol line is higher than the pure lipid line by a factor of 1.3 .

Table II shows that adding cholesterol to DMPC reduces $D_{W}^{\prime}$ considerably. Equation (10) shows that an increase in $K_{\mathrm{C}}$ reduces the $P_{0}$ in the fluctuation pressure in Eq. (11) and this helps bring about the reduction in $D_{W}^{\prime}$. Table II shows a smaller reduction in $D_{W}^{\prime}$ for SOPC, which has a smaller increase in $K_{\mathrm{C}}$, and a small increase in $D_{W}^{\prime}$ for the two dimonounsaturated lipids, which have no increase in $K_{\mathrm{C}}$. Also, as mentioned in the preceding paragraph, adding cholesterol shifts the $B\left(D_{W}^{\prime}\right)$ values which also changes $P_{0}$ according to Eq. (10). It is also possible that cholesterol changes the value of the Hamaker parameter $H$ in Eq. (9). Table II shows values of $H$ that were calculated by balancing the van der Waals pressure in Eq. (9) against the fluctuation pressure extrapolated to the fully hydrated value of $D_{W}^{\prime}$. Although uncertainties in $H$ are large and the present value of $H$ for DMPC with no cholesterol is a factor of 2 greater than a previous value from this laboratory, the values are generally within a factor of 2 of the theoretically acceptable range for lipid bilayers [64]. While cholesterol at $c=0.3$ has negligible effect on $H$ for the unsaturated lipid bilayers, the values in Table II suggest that it may have a non-negligible effect on $H$ for DMPC.

\section{TEMPERATURE DEPENDENCE OF $K_{\mathrm{C}}$}

It is always pleasing from a physical perspective when universal behavior is found, as was reported for the behavior

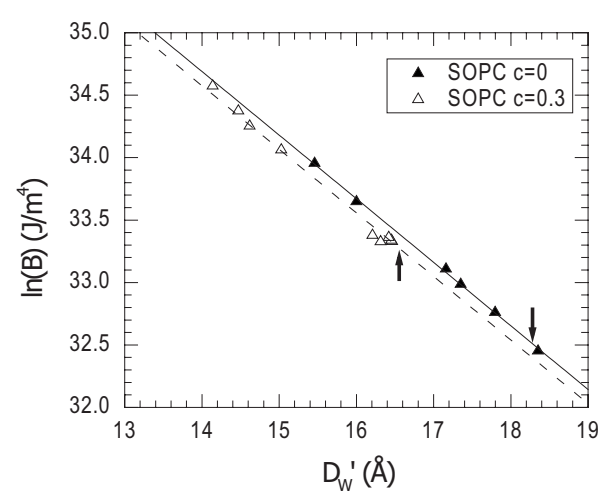

FIG. 10. Log of the bulk compression modulus $B$ versus water spacing $D_{W}^{\prime}$ between neighboring bilayers for SOPC, fitted with the solid straight line, and SOPC with mole fraction $c=0.3$ cholesterol, fitted with the dashed straight line. The arrows indicate the fully hydrated values of $D_{W}^{\prime}$. 
of three similar sterols, cholesterol, lanosterol, and ergosterol, in just one lipid, POPC [15]. In contrast, we previously emphasized that the behavior of cholesterol in different lipids appeared not to be universal [6]. At least, universality appeared to require subclasses that take into account the number of saturated hydrocarbon chains and this raises the possibility that there may be other natural lipid variations, such as having more than one double bond per chain, the location of the double bonds, whether the double bond is cis or trans, and having different headgroups [6]. We next consider whether universality could still be salvaged for the lipids we studied by considering temperature scaling, in particular, using a reduced temperature relative to the chain melting main transition temperature $T_{\mathrm{M}}$. It is well known that the use of reduced temperature is essential to the study of critical points. Although the main transition in lipids has some critical aspects [67], it definitely is a first-order transition in single component lipid bilayers and it is not a priori obvious that reduced temperature is appropriate for scaling first-order transitions.

The main transition temperatures are $T_{\mathrm{M}}=24^{\circ} \mathrm{C}$ for DMPC [68], $T_{\mathrm{M}}=6{ }^{\circ} \mathrm{C}$ for SOPC [69], $T_{\mathrm{M}}=-17^{\circ} \mathrm{C}$ for DOPC [70], and $T_{\mathrm{M}}=13{ }^{\circ} \mathrm{C}$ for diC22:1PC [70]. Our $K_{\mathrm{C}}$ data reported in [6] were obtained at $T=30{ }^{\circ} \mathrm{C}$, so the reduced temperature was the smallest for DMPC and the largest for DOPC, and this corresponds qualitatively to the result that $K_{\mathrm{C}}$ increases most for DMPC with the addition of cholesterol. However, it does not account for diC22:1PC, whose $T_{\mathrm{M}}$ is even larger than for SOPC, and yet $K_{\mathrm{C}}$ for diC22:1PC is not affected by cholesterol, but $K_{\mathrm{C}}$ for SOPC is affected by cholesterol. To further explore the possible implications of reduced temperature, we measured $K_{\mathrm{C}}$ for DMPC, with and without $30 \%$ cholesterol, and diC22:1PC, with and without $40 \%$ cholesterol, at additional temperatures. The results are shown in Fig. 11. At the relative temperature $T-T_{\mathrm{M}}$ of $6{ }^{\circ} \mathrm{C}$, $K_{\mathrm{C}}$ for DMPC/cholesterol is 4.7-fold larger than for DMPC, and at the relative temperature of $24{ }^{\circ} \mathrm{C}, K_{\mathrm{C}}$ for DMPC/ cholesterol is 2.3-fold larger than for DMPC. Similar results for DMPC/cholesterol have been reported [71]. Recalling that $K_{\mathrm{C}}$ for SOPC with $30 \%$ cholesterol is also $\sim 2$-fold larger than for SOPC at a relative temperature of $24^{\circ} \mathrm{C}$, we might be tempted to conclude that the effect of cholesterol on $K_{\mathrm{C}}$ is simply related to the reduced temperature. Contrarily, even at a relative temperature of $1.7^{\circ} \mathrm{C}$, diC22:1PC with $c$ $=0.4$ cholesterol has essentially the same $K_{\mathrm{C}}$ as diC22:1PC. A strong temperature dependence of the elastic modulus $K_{\mathrm{A}}$ for $\mathrm{SOPC} /$ cholesterol and a weaker temperature dependence for DOPC/cholesterol have also been reported [7]. The conclusion is that, even though the reduced temperature should play a role in discussing the behavior of $K_{\mathrm{C}}$ as a function of cholesterol concentration, a necessary feature is still the number of saturated chains, and many other possible subclasses of universality may ensue from other variations of lipid molecules.

\section{GENERAL DISCUSSION}

While the conclusion of the previous section, that universality may not be particularly useful, is not appealing physi-

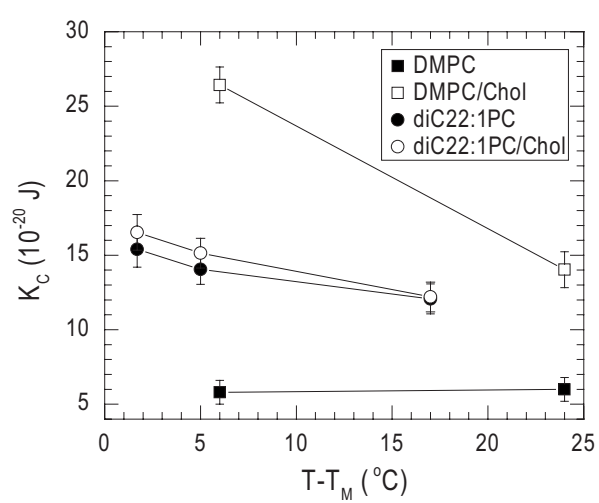

FIG. 11. Bending modulus $K_{\mathrm{C}}$ versus relative temperature $T-T_{\mathrm{M}}$ where the melting temperature $T_{\mathrm{M}}$ for DMPC and DMPC/ cholesterol is $24{ }^{\circ} \mathrm{C}$ and for diC22:1PC and diC22:1PC/cholesterol is $13{ }^{\circ} \mathrm{C}$. The cholesterol mole fraction was $c=0.3$ for the DMPC/ cholesterol mixture and was $c=0.4$ for the $\mathrm{diC} 22: 1 \mathrm{PC} /$ cholesterol mixture.

cally, it has biological appeal. There are many reasons for mammalian membranes to contain considerable amounts of cholesterol. However, if membranes contained only saturated lipids, cholesterol would make them less flexible by increasing $K_{\mathrm{C}}$ and that could only make more difficult the many shape changes required by living cells. Replacing saturated lipid chains with unsaturated chains mitigates this undesirable effect of cholesterol. While this could hypothetically be accomplished within the context of a universal theory that involves the reduced temperature, our experimental results suggest instead that it involves detailed physical interactions imposed by the chemical specificity of the lipid molecules.

Section V of the present paper emphasizes that new ideas are required to understand how cholesterol affects the material moduli $K_{\mathrm{C}}$ and $K_{\mathrm{A}}$. We present a theory due to Evans that appears to be on the right track, although it accounts only for high cholesterol concentrations. As such, it does not explicitly include differences in the interaction of cholesterol with lipids having different numbers of saturated chains.

Regarding interactions, it has been well recognized that cholesterol has a smooth face and a rough face due to two bulky methyls sticking out on the same rough side of the otherwise planar ring structure [2-5]. Therefore, saturated chains are more likely to pack against the smooth face, which then acts to condense them into straighter chains taking up considerably less area. $C$ is unsaturated chains would therefore have weaker interactions with cholesterol and would not be as susceptible to condensing. Our results for the partial molecular area of cholesterol dramatically exhibit this difference in the condensing effect. Our results for the bilayer thickness $D_{\mathrm{HH}}$ and the order parameter $S_{\text {xray }}$ are consistent with the straightening of the chains and support the conventional wisdom that cholesterol increases orientational order while the width of our wide angle peaks confirms the relative lack of lateral packing order. Our results for $S_{\text {xray }}$ in Fig. 7 show that equal concentrations of cholesterol increase $S_{\text {xray }}$ more for saturated chains than for unsaturated chains, which supports different interaction strengths with cholesterol [4].

The phenomenological model of noninteracting clusters of cholesterol plus lipid [50] embodies the interaction 
strength by the number of lipids $p$ in each cluster. The fits to our data in Fig. 7 obtain values of $p$ twice as large for lipids with saturated chains, which is consistent with stronger interactions of saturated chains with cholesterol. However, our values of $p[6]$ assumed that there is only one $(q=1)$ cholesterol in each cluster. One might also consider the possibility that the smooth face of cholesterol not only interacts favorably with saturated chains, but that it also interacts unfavorably with unsaturated chains. This would provide an effective interaction in dimonounsaturated bilayers that would favor dimers of cholesterol with the two smooth faces facing each other. This would double $q$ and also $p$ in the cluster model, so the number of lipids in a cluster in dimonounsaturated bilayers would be the same as for saturated lipids but the doubled $q$ would lead to the doubled values of $c$ where the leveling off of $S_{\text {xray }}$ occurs in Fig. 7.

Our x-ray data do not directly provide the lateral organization of cholesterol in lipid bilayers, even with the extensive interpretation employed in this paper. However, we suggest that a way forward would compare atomically detailed simulations with primary $\mathrm{x}$-ray scattering data that consist of both LAXS $\left|F\left(q_{z}\right)\right|$ data in Fig. 1 and extensive twodimensional WAXS data [23]. Atomic level simulations cannot generally be run long enough to allow much lateral dif- fusion to provide lateral equilibration. However, one initial state could include configurations like the dimer configuration mentioned above and another initial state could include a random distribution of cholesterol, and a third could include a more regular array of cholesterol consistent with effectively repulsive cholesterol-cholesterol interactions [72]. The best agreement with the primary x-ray data would then favor that state.

\section{ACKNOWLEDGMENTS}

We thank Evan Evans for elucidating the model for the high cholesterol limit and giving permission to quote $K_{\mathrm{A}}$ data before publication and Ben Smith for first showing us these data. We acknowledge Thalia Mills for helping to acquire and analyze some of the data originally reported [6]. Many of the x-ray scattering data were taken at the Cornell High Energy Synchrotron Source (CHESS), which is supported by the National Science Foundation and the National Institutes of Health/National Institute of General Medical Sciences under National Science Foundation Grant No. DMR-0225180. This research was supported by NIH Institute of General Medical Sciences Grant No. GM44976 (PI-JFN).
[1] B. Alberts, D. Bray, J. Lewis, M. Raff, K. Roberts, and J. D. Watson, Molecular Biology of the Cell (Garland Publishing, Inc., New York, 1994).

[2] G. W. Feigenson, Biochim. Biophys. Acta 1788, 47 (2009).

[3] T. P. W. McMullen, R. N. A. H. Lewis, and R. N. McElhaney, Curr. Opin. Colloid Interface Sci. 8, 459 (2004).

[4] J. R. Silvius, Biochim. Biophys. Acta 1610, 174 (2003).

[5] M. L. Berkowitz, Biochim. Biophys. Acta 1788, 86 (2009).

[6] J. J. Pan, T. T. Mills, S. Tristram-Nagle, and J. F. Nagle, Phys. Rev. Lett. 100, 198103 (2008).

[7] W. Rawicz, B. A. Smith, T. J. Mclntosh, S. A. Simon, and E. Evans, Biophys. J. 94, 4725 (2008).

[8] J. B. Finean, Chem. Phys. Lipids 49, 265 (1989).

[9] J. B. Finean and A. L. Hutchinson, Chem. Phys. Lipids 46, 63 (1988).

[10] S. Mabrey, P. L. Mateo, and J. M. Sturtevant, Biochemistry 17, 2464 (1978).

[11] P. J. Davis and K. M. W. Keough, Biochemistry 22, 6334 (1983).

[12] M. Pasenkiewicz-Gierula, W. K. Subczynski, and A. Kusumi, Biochemistry 29, 4059 (1990).

[13] M. R. Ali, K. H. Cheng, and J. Y. Huang, Proc. Natl. Acad. Sci. U.S.A. 104, 5372 (2007).

[14] T. P. W. McMullen and R. N. McElhaney, Biochemistry 36, 4979 (1997).

[15] J. Henriksen, A. C. Rowat, E. Brief, Y. W. Hsueh, J. L. Thewalt, M. J. Zuckermann, and J. H. Ipsen, Biophys. J. 90, 1639 (2006).

[16] N. Kučerka, S. Tristram-Nagle, and J. F. Nagle, J. Membr. Biol. 208, 193 (2005).

[17] N. Kučerka, J. Pencer, J. N. Sachs, J. F. Nagle, and J. Katsaras,
Langmuir 23, 1292 (2007).

[18] S. Tristram-Nagle, in Methods in Molecular Biology, edited by A. M. Dopico (Humana Press, Totowa, NJ, 2007), Vol. 400, p. 63.

[19] Y. Lyatskaya, Y. F. Liu, S. Tristram-Nagle, J. Katsaras, and J. F. Nagle, Phys. Rev. E 63, 011907 (2001).

[20] Y. F. Liu and J. F. Nagle, Phys. Rev. E 69, 040901 (2004).

[21] J. F. Nagle and S. Tristram-Nagle, Biochim. Biophys. Acta 1469, 159 (2000).

[22] N. Kučerka, J. F. Nagle, S. E. Feller, and P. Balgavý, Phys. Rev. E 69, 051903 (2004).

[23] T. T. Mills, G. E. S. Toombes, S. Tristram-Nagle, D. M. Smilgies, G. W. Feigenson, and J. F. Nagle, Biophys. J. 95, 669 (2008).

[24] J. B. Klauda, N. Kučerka, B. R. Brooks, R. W. Pastor, and J. F. Nagle, Biophys. J. 90, 2796 (2006).

[25] A. I. Greenwood, S. Tristram-Nagle, and J. F. Nagle, Chem. Phys. Lipids 143, 1 (2006).

[26] J. F. Nagle and M. C. Wiener, Biochim. Biophys. Acta 942, 1 (1988).

[27] S. Tristram-Nagle, Y. Liu, J. Legleiter, and J. F. Nagle, Biophys. J. 83, 3324 (2002).

[28] S. A. Pandit, S. W. Chiu, E. Jakobsson, A. Grama, and H. L. Scott, Langmuir 24, 6858 (2008).

[29] H. Martinez-Seara, T. Róg, M. Karttunen, R. Reigada, and I. Vattulainen, J. Chem. Phys. 129, 105103 (2008).

[30] C. J. Högberg, A. M. Nikitin, and A. P. Lyubartsev, J. Comput. Chem. 29, 2359 (2008).

[31] S. W. Chiu, S. A. Pandit, H. L. Scott, and E. Jakobsson, J. Phys. Chem. B 113, 2748 (2009).

[32] N. Kučerka, J. D. Perlmutter, J. Pan, S. Tristram-Nagle, J. 
Katsaras, and J. N. Sachs, Biophys. J. 95, 2792 (2008).

[33] W. C. Hung, M. T. Lee, F. Y. Chen, and H. W. Huang, Biophys. J. 92, 3960 (2007).

[34] J. Gallová, D. Uhríková, M. Hanulová, J. Teixeira, and P. Balgavý, Colloids Surf., B 38, 11 (2004).

[35] J. Pencer, M. P. Nieh, T. A. Harroun, S. Krueger, C. Adams, and J. Katsaras, Biochim. Biophys. Acta 1720, 84 (2005).

[36] N. Kučerka, J. Pencer, M. P. Nieh, and J. Katsaras, Eur. Phys. J. E 23, 247 (2007).

[37] M. Gandhavadi, D. Allende, A. Vidal, S. A. Simon, and T. J. McIntosh, Biophys. J. 82, 1469 (2002).

[38] N. Kučerka, J. F. Nagle, J. N. Sachs, S. E. Feller, J. Pencer, A. Jackson, and J. Katsaras, Biophys. J. 95, 2356 (2008).

[39] O. Edholm and J. F. Nagle, Biophys. J. 89, 1827 (2005).

[40] B. M. Craven, Nature (London) 260, 727 (1976).

[41] S. W. Hui and N. B. He, Biochemistry 22, 1159 (1983).

[42] T. H. Huang, C. W. B. Lee, S. K. Dasgupta, A. Blume, and R. G. Griffin, Biochemistry 32, 13277 (1993).

[43] J. H. Ipsen, O. G. Mouritsen, and M. Bloom, Biophys. J. 57, 405 (1990).

[44] M. R. Vist and J. H. Davis, Biochemistry 29, 451 (1990).

[45] C. Hofsäß, E. Lindahl, and O. Edholm, Biophys. J. 84, 2192 (2003).

[46] J. Y. Huang, J. T. Buboltz, and G. W. Feigenson, Biochim. Biophys. Acta 1417, 89 (1999).

[47] Y. W. Hsueh, K. Gilbert, C. Trandum, M. Zuckermann, and J. Thewalt, Biophys. J. 88, 1799 (2005).

[48] Y. W. Hsueh, M. T. Chen, P. J. Patty, C. Code, J. Cheng, B. J. Frisken, M. Zuckermann, and J. Thewalt, Biophys. J. 92, 1606 (2007).

[49] J. A. Urbina, S. Pekerar, H. B. Le, J. Patterson, B. Montez, and E. Oldfield, Biochim. Biophys. Acta 1238, 163 (1995).

[50] H. McConnell and A. Radhakrishnan, Proc. Natl. Acad. Sci. U.S.A. 103, 1184 (2006).

[51] M. R. Brzustowicz, V. Cherezov, M. Caffrey, W. Stillwell, and S. R. Wassall, Biophys. J. 82, 285 (2002).

[52] S. L. Niu and B. J. Litman, Biophys. J. 83, 3408 (2002).

[53] F. A. Nezil and M. Bloom, Biophys. J. 61, 1176 (1992).

[54] H. Schindler and J. Seelig, Biochemistry 14, 2283 (1975).

[55] A. Seelig and J. Seelig, Biochemistry 13, 4839 (1974).
[56] J. B. Song and R. E. Waugh, Biophys. J. 64, 1967 (1993).

[57] B. Sorre, A. Callan-Jones, J. B. Manneville, P. Nassoy, J. F. Joanny, J. Prost, B. Goud, and P. Bassereau, Proc. Natl. Acad. Sci. U.S.A. 106, 5622 (2009).

[58] A. W. Tian, B. R. Capraro, C. Esposito, and T. Baumgart, Biophys. J. (in press).

[59] R. S. Gracia, N. Bezylyepkina, R. L. Knorr, R. Lipowsky, and R. Dimova, Soft Matter (in press).

[60] W. Rawicz, K. C. Olbrich, T. McIntosh, D. Needham, and E. Evans, Biophys. J. 79, 328 (2000).

[61] J. Pan, S. Tristram-Nagle, N. Kučerka, and J. F. Nagle, Biophys. J. 94, 117 (2008).

[62] E. Evans and W. Rawicz, Phys. Rev. Lett. 64, 2094 (1990).

[63] A. Yeung and E. Evans, J. Phys. II 5, 1501 (1995).

[64] H. I. Petrache, N. Gouliaev, S. Tristram-Nagle, R. T. Zhang, R. M. Suter, and J. F. Nagle, Phys. Rev. E 57, 7014 (1998).

[65] S. Tristram-Nagle and J. F. Nagle, Biophys. J. 93, 2048 (2007).

[66] R. Podgornik and V. A. Parsegian, Langmuir 8, 557 (1992).

[67] N. Chu, N. Kucerka, Y. F. Liu, S. Tristram-Nagle, and J. F. Nagle, Phys. Rev. E 71, 041904 (2005).

[68] R. N. A. H. Lewis, N. Mak, and R. N. McElhaney, Biochemistry 26, 6118 (1987).

[69] C. Vilchèze, T. P. W. McMullen, R. N. McElhaney, and R. Bittman, Biochim. Biophys. Acta 1279, 235 (1996).

[70] R. N. A. H. Lewis, B. D. Sykes, and R. N. McElhaney, Biochemistry 27, 880 (1988).

[71] P. Méléard, C. Gerbeaud, T. Pott, L. Fernandez-Puente, I. Bivas, M. D. Mitov, J. Dufourcq, and P. Bothorel, Biophys. J. 72, 2616 (1997)

[72] J. Y. Huang and G. W. Feigenson, Biophys. J. 76, 2142 (1999).

[73] B. A. Smith, T. J. McIntosh, and E. A. Evans (unpublished).

[74] Z. Chen and R. P. Rand, Biophys. J. 73, 267 (1997).

[75] N. Kučerka, Y. F. Liu, N. J. Chu, H. I. Petrache, S. TristramNagle, and J. F. Nagle, Biophys. J. 88, 2626 (2005).

[76] A. I. Greenwood, J. J. Pan, T. T. Mills, J. F. Nagle, R. M. Epand, and S. Tristram-Nagle, Biochim. Biophys. Acta 1778, 1120 (2008).

[77] J. C. Mathai, S. Tristram-Nagle, J. F. Nagle, and M. L. Zeidel, J. Gen. Physiol. 131, 69 (2008). 